\title{
Using Twitter to Learn about the Autism Community
}

\author{
Adham Beykikhoshk, Ognjen Arandjelović, \\ Dinh Phung, Svetha Venkatesh, and Terry Caelli
}

the date of receipt and acceptance should be inserted later

\begin{abstract}
Considering the raising socio-economic burden of autism spectrum disorder (ASD), timely and evidence-driven public policy decision making and communication of the latest guidelines pertaining to the treatment and management of the disorder is crucial. Yet evidence suggests that policy makers and medical practitioners do not always have a good understanding of the practices and relevant beliefs of ASD-afflicted individuals' carers who often follow questionable recommendations and adopt advice poorly supported by scientific data. The key goal of the present work is to explore the idea that Twitter, as a highly popular platform for information exchange, could be used as a data-mining source to learn about the population affected by ASD - their behaviour, concerns, needs etc. To this end, using a large data set of over 11 million harvested tweets as the basis for our investigation, we describe a series of experiments which examine a range of linguistic and semantic aspects of messages posted by individuals interested in ASD. Our findings, the first of their nature in the published scientific literature, strongly motivate additional research on this topic and present a methodological basis for further work.
\end{abstract}

Centre for Pattern Recognition and Data Analytics (PRaDA)

School of Information Technology

Deaking University

Waurn Ponds Geelong 3216

VIC

Australia 
Keywords Social media $\cdot$ Big Data $\cdot$ Asperger's $\cdot$ Mental health $\cdot$ Health care $\cdot$ Public health $\cdot$ ASD

\section{Introduction}

In this paper we are interested in leveraging the remarkable increase in the use of social media, and Twitter in particular, to obtain information about specific demographic communities which are difficult to reach by conventional means. To illustrate our ideas and demonstrate their effectiveness, herein we focus on the population affected by the autism spectrum disorder (ASD) - a neurodevelopmental disorder which has been attracting increasing attention as much for its complex and varied aetiology, as for the associated and rapidly growing socioeconomic burden. The population of interest in this work includes both individuals who have been diagnosed with ASD themselves, as well as those who are indirectly but significantly impacted such as the family members and carers of ASD sufferers. To the best of our knowledge ours is the first work of this nature. The present paper builds on the preliminary results reported in [1] and describes a number of novel findings and experiments whose results further support the key underlying idea and motivate additional work in this direction. Most notable novelties include:

- additional statistics of the collected tweet corpus,

- quantitative analysis of Zipf's law for tweets and ASD-related tweets in particular,

- examination of statistical significance of differential part-of-speech characteristics,

- experiments using least absolute shrinkage and selection operator (LASSO),

- additional evidence of content saliency using an Alzheimer's disease tweet corpus, and

- new analysis of the procedure for the selection of the bootstrap keyword set. 
It should also be noted that the results reported in this paper include a significantly expanded corpus of tweets (nearly an order of magnitude greater) as we continued collecting data following the publication of our original work.

The remainder of this paper is structured as follows. In the next section we describe some of the key aspects of the autism spectrum disorder which motivated our focus on this particular condition. In Section 3 we review relevant previous work on the use of Twitter for data mining and highlight the key techniques and methods in the field. Section 4 describes the main contribution of our work - we start by describing the manner in which the data used for the present study was collected and follow up with a description, results, and discussion of a sequence of increasingly complex experiments. A summary of the work and its findings is made in Section 5

\section{Motivation and relevant background}

Autism spectrum disorder is a life-long neurodevelopmental disorder with poorly understood causes on the one hand, and a wide range of potential treatments supported by little evidence on the other. The disorder is characterized by severe impairments in social interaction, communication, and in some cases cognitive abilities, and typically begins in infancy or at the very latest by the age of three. ASD is recognized as comprising an aetiologically and clinically heterogeneous group of conditions whose diagnosis remains to be based solely on the complex behavioural phenotype [2]. According to the definition in the latest version (5th edition) of the Diagnostic and Statistical Manual of Mental Disorders, the autism spectrum disorder includes disorders which were previously diagnosed with more specificity as autism, Asperger syndrome, Rett syndrome, childhood disintegrative disorder, and 'pervasive developmental disorder not otherwise specified' [3]. Current evidence suggests that approximately $0.5-0.6 \%$ of the population is afflicted by ASD though the actual diagnosis rate is on the increase due to the broadening diagnostic criteria [4]. The condition is usually detected in early childhood when an abnormal lack of social reciprocity is observed.

Considering the social and economic burden of ASD it is unsurprising that it has been attracting an increasing amount of research attention; numerous longitudinal, epidemiolog- 
ical, and family studies have been conducted [5]. This is illustrated by the plot in Fig. 1 which shows the number of academic papers in English with the word "autism" in the title or abstract (as indexed by the PubMed portal which interfaces the US National Library of Medicine life and biomedical sciences database). The earliest work is that by Kanner in 1946 [6] with a readily observed exponential increase thenceforth. Note that the count for the year 2014, which seemingly bucks the trend, is in fact perfectly in agreement with the overall increase as only papers indexed by PubMed up to and including May 2014 are included.

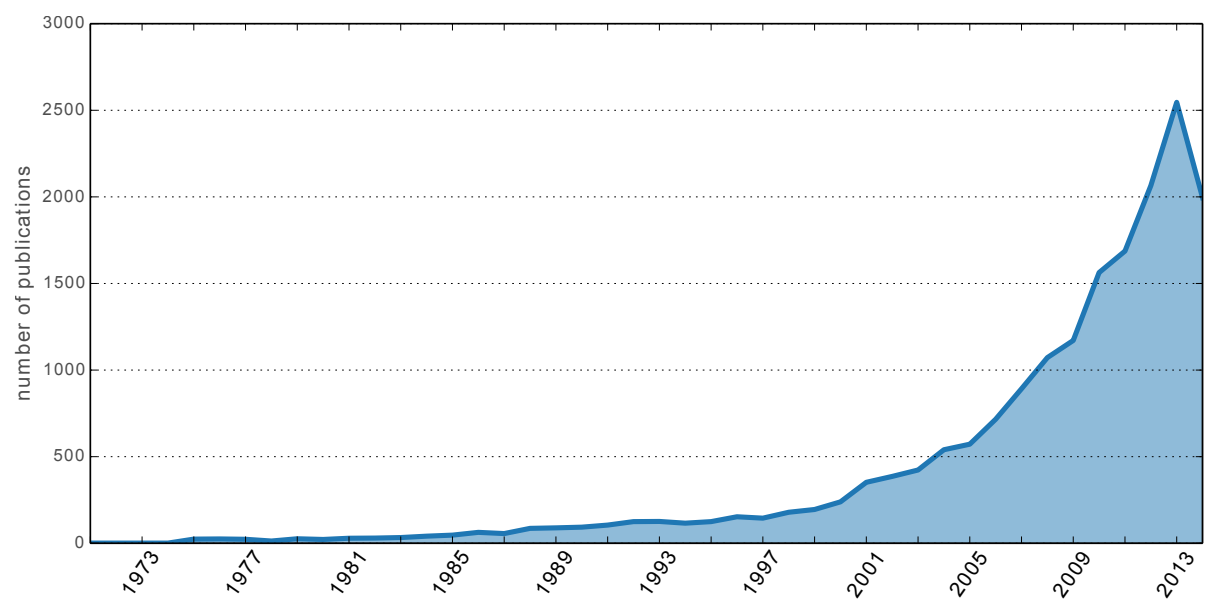

Fig. 1: The number of academic papers in English with the word "autism" in the title or abstract as indexed by the PubMed portal which interfaces the US National Library of Medicine life and biomedical sciences database. The earliest work is that by Kanner in 1946 [6]; thereafter an exponential increase is readily observed. Note that the count for 2014 is incomplete as it includes papers indexed by PubMed only up to and including May 2014.

Although the last few decades have seen significant progress in the study of ASD, the still relatively poorly understood aetiology of the condition, its phenotypical heterogeneity [7], and stigma associated with mental conditions [8], have all contributed to the penetration of beliefs, and behavioural and educational interventions which are often ques- 
tionable [9] and poorly supported by evidence (e.g. gluten-free and casein-free diets, and cognitive behavioural therapy [10]), and sometimes outright in conflict with science [11]. For example a recent review of early intensive behavioural and developmental interventions for young children with ASD found 1 existing study as being of good quality, 10 as fair quality, and 23 as poor quality [9]. From the public policy point of view, understanding the practices and beliefs of parents and carers of ASD-affected individuals is crucial, yet often lacking [12].

Thus the key idea motivating the present work is that the rapid rise in the adoption of social media as a platform for the expression and exchange of ideas, which facilitates the emergence of special interest communities, can be used to study and monitor the beliefs and practices of the population affected by ASD. Considering the challenge of reaching and engaging with this specific target population, our findings pave the way for further work of potential significant benefit to public health. These benefits include the enrichment of the corpus of knowledge of the condition itself by the medical community, and the increased understanding of the practices and concerns of those affected by ASD.

\section{Previous work}

While this is the first work exploring the possibility of using Twitter data for the extraction of ASD-related information, the broad idea of data-mining Twitter is not new and has been employed successfully in a variety of applications. At the same time it is important to stress at the very outset that most of the previous work on this topic has not been automatic, that is, analysis was performed 'manually' by humans. This is a laborious process which severely limits how much data can be processed. Additionally, the use of human intelligence rather than computer-based methods means that in the case of many reported results, it is not clear that the same results could be obtained automatically due to a possible semantic gap.

A popular research direction focuses on various forms of prediction based on tweet sentiments [13] inferred from the tweet's emoticons [14] or using linguistics-based classifiers [15]. For example, Asur and Huberman [16] showed that tweet posting rate can be used to forecast film box office revenues, while Baucom et al. [17] used sentiment to analyse the 
relationship between the content of tweets and the outcomes of NBA Playoff games. Bollen et al. [18] showed that tweet sentiments, aggregately seen as a reflection of public mood levels, can be used to predict the incidence of socio-political, cultural, and economic events. Mitchell et al. [19] used geo-tagged tweets across the United States to estimate localized changes in a variety of sociometric indices such as happiness, the level of education, and obesity rates.

The quasi-realtime nature of Twitter also makes it a potentially valuable resource for the detection and management of emergency situations [20]. For example, Robinson et al. [21] described an earthquake detection system, while Sakaki et al. [22] used a spatio-temporal model of tweet frequencies to infer the location of the epicentre of an earthquake.

Closer in spirit to the nature of work in the present paper is the corpus of work on the use of Twitter in the domain of health care. For example, Paul and Dredze [23] used tweets to extract words related to symptoms and treatments, and a topic model to associate them with the corresponding ailments. In their subsequent work [24], the model was extended to track the spread of illnesses over time, measure behavioural risk factors, and analyse symptoms and medication usage. The use of LDA-based health topic modelling was explored by Prier et al. [25]. Dementia-related tweets were the focus of work by Robillard et al. [26] who collected relevant tweets over a $24 \mathrm{~h}$ period using keyword filtering and used them to discover the dominant chatter themes. Similarly, Scanfeld et al. [27] used Twitter to analyse the patterns of antibiotic use. Jashnisky et al. [28] studied the relationship between Twitter conversations deemed to reflect high suicide risk and actual suicide rates in the United States. They demonstrated that high risk individuals may be recognized from their social media status. Lastly, Himelboim and Han [29] examined the connectivity patterns of Twitter users interacting within a specific online community of cancer-affected individuals.

In contrast to the sporadic studies on different diseases described above, the use of Twitter data in the management of highly contagious diseases like influenza has attracted a more concentrated research effort. For example, Culotta [30] investigated whether the frequency of influenza epidemic-related tweets can be related to 'ground truth' data from centres for disease control and prediction. Achrekar et al. [31] showed that the emergence and the 
spread of epidemic influenza can be predicted and tracked from the location and demographic information of users of relevant tweets. A similar approach was also described by $\mathrm{Li}$ and Cardie [32]. Yet further evidence of the power of Twitter data was presented by Chew and Eysenbach [33] who demonstrated that the spatio-temporal distribution of relevant tweet frequencies during the $2009 \mathrm{H} 1 \mathrm{~N} 1$ outbreak closely matches the disease spreading pattern.

Although the use of Twitter for data-mining information related to ASD has not been explored yet, there has been some preliminary work on the use of other social media and ASD. For example, Newton et al. [34] used Linguistic Inquiry and Word Count (LIWC) dictionaries to compare writing patterns of individuals with ASD and those of neuro-typical bloggers.

\section{Methods, results, and discussion}

Having outlined the motivation and our ultimate vision for this work, and placed it in context of previous research on data-mining social media, we now turn our attention to the main contribution of the present paper. We start by describing the data set we used for our analysis.

\subsection{Data acquisition}

Twitter's Terms of Service explicitly prohibit the sharing or redistribution of tweets, including for research purposes. Consequently, there was no public data set that we could use as a standard benchmark in this study. Instead, we collected a large data set ourselves.

Twitter API offers different means of retrieving tweets. In particular, we used its 'search' and 'streaming' functions. The former allows the retrieval of historical tweets based on the presence of specific keywords and meta-data constraints (e.g. on the language or user location). After being posted, a tweet can be obtained in this manner for up to a week. The streaming API allows a quasi-realtime retrieval of tweets as they are posted, retrieving a sample of approximately $1 \%$ of all tweets. The search API was most valuable for us for collecting ASD-related content, as we will describe in detail shortly. Conversely, the streaming 
API allowed us to obtain 'control' data, unrelated to ASD, since this set could not be well characterized a priori using a compact set of keywords.

We collected only tweets posted in English. To facilitate a comparative analysis, we collected two non-overlapping data sets. The first of these, which we will refer to as the ASD subset, comprises tweets which concern ASD. Specifically, we defined the ASD subset as comprising tweets which contain any of the four keywords "autism", "adhd", "asperger", and "aspie" (or any of their derivatives obtained by suffixation), and the control subset as comprising all other tweets. In total this resulted in a corpus of 5,650,989 ASD-related tweets collected in the period starting on 26 August 2013 and ending on 1 Oct 2014 (i.e. more than 13 consecutive months). Of these, 3,493,742 were original tweets which were produced by $1,771,274$ unique users, with the remaining $28 \%$ of the messages being retweets. Approximately $25 \%$ of the collected tweets $(2,260,284)$ contain so-called hashtags (see Section 4.4.2 for discussion and analysis). Although this information was not used in the present work we also report that in our corpus 70,925 tweets are geo-tagged (it should be noted that geo-tags, being controlled entirely by the user, are not necessarily correct location identifiers), 2,599,395 contain URLs, 464,190 were sent in reply, and 3,330,096 mention another user.

It is important to observe that it is not our claim that tweets in the ASD subset were necessarily posted by individuals suffering from ASD. While some of the messages in this subset do fall into this category, the subset will also include posts by individuals affected by ASD in a looser sense, e.g. parents or carers of those who suffer from ASD, or indeed medical professionals interested in the condition.

\subsubsection{Pre-processing}

Much of the work in the present paper concerns the analysis of topics discussed by means of Twitter. In this context it is beneficial to have different inflections of the same word normalized and represented by a single term. In linguistics this process is referred to as lemmatization and we apply it automatically using the freely available Natural Language Toolkit (NLTK) [35]. In addition, we remove the so-called 'stop words' which do not carry 


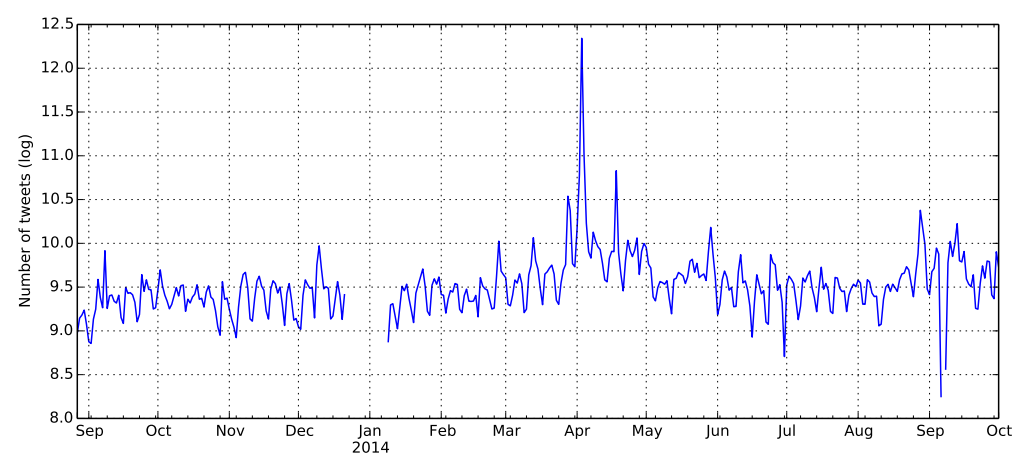

Fig. 2: Number of ASD-related tweets we collected each day over the course of our data acquisition period; notice weekly periodicity. To make the usual day to day variability visible in the presence of the sharp peak on 2 April (World Autism Awareness Day) logarithmic scaling was used for ordinate values. The approximately three week gap in data collection during the 2014-2015 holiday period was caused by the failure of our computer system.

much meaning themselves (e.g. articles and connectives), as well as all punctuation marks and emoticons. To illustrate the effects of our pre-processing we present a few examples.

\section{Original tweet:}

Looks like we will have more \#autism research happening for children in \#EarlyIntervention next year! :-) \#VisualSupports \#MobileTech

\section{Pre-processed result:}

look like autism research happen child earlyintervention visualsupports mobiletech

\section{Original tweet:}

Authors who see autism as "tremendously burdening" elicit dire views of autism from parents http://j.mp/lFyqXwF "Ethical approval: none"

\section{Pre-processed result:}

author see autism tremend burden elicit dire view autism parent url ethic approv none 


\section{Original tweet:}

101 autism: Genetic analysis of individuals with autism finds gene deletions - Using powerful genetic sequencing. http://is.gd/UhprQK

\section{Pre-processed result:}

101 autism genet analysi individu autism find gene delet use power genet sequenc

url

\section{Original tweet:}

\#Apple \#Censorship \& Dr. Brian Hooker Interview exposing CDC Cover-up of the Vaccine \& Autism Link on . @ rediceradio http://youtu.be/19uvPtg6SPI

\section{Pre-processed result:}

appl censorship dr brian hooker interview expos cdc cover vaccin autism link atus url

\subsection{Methods and results}

In this section we describe a series of experiments aimed at discovering the properties of the collected data. We start with a generic quantitative linguistic analysis and proceed with increasingly domain-specific considerations which examine tweet content and the potential differentiation between the ASD-related and the control corpora.

\subsubsection{Power law: Zipf's distribution}

The first experiment we conducted was set to find out if tweets, both in the ASD corpus as well as the control group, obey the so-called Zipf's law. In its general form, this empirical law posits that the frequency $P_{i}$ of an 'event' is approximately a power function of its frequency rank $r_{i}$, i.e. $P_{i} \propto r_{i}^{a}$ where $r_{i}$ is the rank $\left(r_{i}=1, \ldots\right)$ of the event when events are ordered by their frequency of occurrence from high to low and $a<-1$. Zipf's distribution can be seen to be a type of a power law probability distribution. This statistical regularity is observed in a variety of domains ranging from deadly quarrel [36] and wealth distribution 
analysis [37], to information retrieval [38] and quantitative linguistics [38]. In quantitative linguistics, an event corresponds to the usage of a particular word or, more precisely in the context of the present paper, a particular term resulting from the pre-processing of a word as described in Section 4.1 .1

Evidence from previous work suggests that conventional texts, such as newspapers or books, do result in Zipfian distributions of word frequencies [38]. However, it is not clear from this that the same applies to tweets. Firstly, tweets are restricted in length to 140 characters which by itself may alter linguistic characteristics of posted messages. In addition, the nature of Twitter as a communication medium invariably introduces a self-selecting aspect: neither can the corpus of Twitter users be considered to be a random sample from the population, nor can the memetic content of tweets be expected to match that of texts examined by previous work, such as newspapers and books.

In our data set, the ASD corpus of tweets contains 47,048,097 terms (pre-processed words) of which 402,946 are unique; the control subgroup used 32,846,321 terms of which 528,755 are unique. The key findings are summarized in Fig. 3(a) which shows the plot of tweet term frequency as a function of its overall term frequency rank. It is readily apparent that the characteristics of both the ASD and the control group are nearly identical. What is more, both can be seen to exhibit approximately linear behaviour on this plot; a mild deviation from this behaviour can be observed for the most frequent terms. There are two main reasons for this. Firstly, it is generally the case that the simple functional form of Zipf's law fails to be observed for the top-ranking events. Secondly, our use of the logarithmic scale for the abscissa means that the left-hand tail of the graph is sparsely populated by data points which increases the corresponding error margins in this plot.

To quantify the linearity of dependence plotted in Fig. 3(a) we used Pearson's $r$ statistic. Specifically, we computed the value of the statistic first for the window which spans $20 \%$ of the ranks on the logarithmic scale and is centred at the logarithmic median of the ranks (i.e. the centre of the plot in Fig. 3(a), and then proceeded to increase the width recomputing the value for the encompassed range until all ranks were included. The variation of Pearson's $r$ as a function of the window width is plotted in Fig. 3(b) confirming our previous observa- 
tions. The linearity of the central portion of the distribution is nearly perfect as witnessed by the $r$ value of 0.999 . As more data is added a slight non-linearity is observed, the statistic dropping down to approximately 0.992 for the entire range.

In addition to their novelty, these findings are interesting in terms of directing our research towards our ultimate goal of automatically retrieving and analysing the content of ASD-related tweets. In particular, the discovery that tweets, including those in the sub-group of our primary interest, obey Zipf's law suggests that it is sensible to adopt and explore the use of a broad range of well-known and well-understood text representations and methods of analysis. Indeed we do this next.

4.3 Message length

Having established that Twitter messages conform to some of the same general linguistic rules as conventional texts do, our next goal was to explore any differential characteristics exhibited by our ASD and control groups. Recall from Section 4.1 that our data set is balanced in the number of tweets, that is, the number of tweets in the ASD corpus is the same as in the control corpus. Yet, as pointed out in the previous section, the term counts in the two data sets are different, respectively 47,048,097 and 32,846,321 - a significant difference of approximately $43 \%$. It is a direct implication of this observation that the average term count per tweet is greater in the ASD group, which is the first indication of there being a difference between tweets in this group and the remainder of our data corpus. The tendency of this group to post longer messages is further corroborated by comparing the corresponding histograms of tweet word counts, which are shown in Fig. 4 . While the tweet word count in both sub-groups can be seen to be log-normally distributed, the two distributions have substantially different means $(p<0.01)$. 


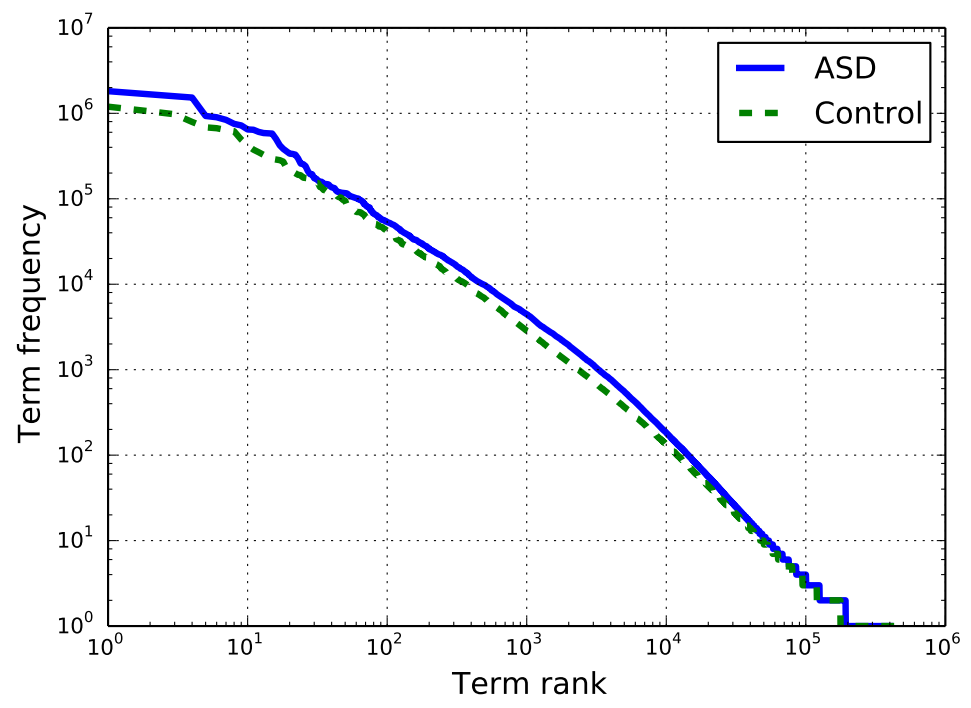

(a)

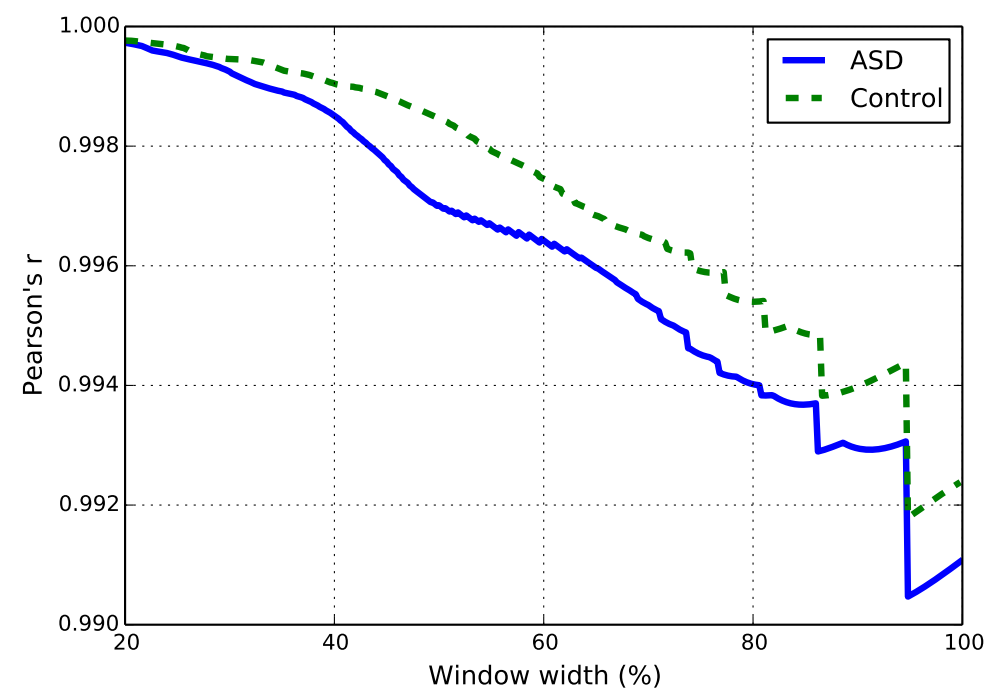

(b)

Fig. 3: (a) A log-log plot showing the dependence of tweet term (word) frequency on its frequency rank within the corpus of all terms in our data set. Approximately linear dependency is exhibited by data extracted from both the ASD and the control data sets, in agreement with the Zipfian power law distribution. (b) Linear dependency is quantified using the Pearson's $r$ statistic. To account for the expected artefacts at the ends of the distribution, the statistic is plotted as a function of the width of the window centred at the logarithmically median rank and spanning $20-100 \%$ of the logarithmic range of possible ranks. Pearson's $r$ attains values of over 0.995 for the central portion of the distribution, and drops slightly (to approximately 0.992) for the entire range when the end artefacts are included. 


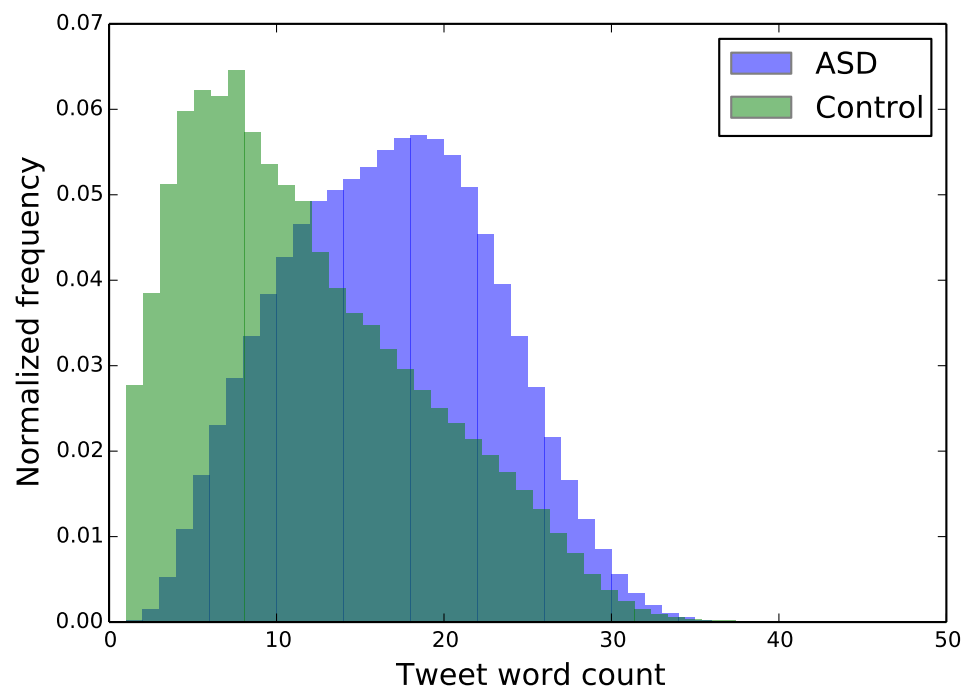

Fig. 4: Normalized histograms of tweet word counts for the two subsets. As expected, lognormal distributions are observed but, importantly in the context of the present work, with different parameters (most obviously the means, $p<0.01$ ).

\subsection{Content analysis}

Our next aim was to explore if the collected data offers evidence that tweets in the ASD and control data sets differ significantly by their content (i.e. topic of conversation) and if so, if automatic methods could be employed in the analysis of this content.

\subsubsection{Word frequency}

In the first experiment we approached this task by comparing the most frequently used words in the two data sets. These words can be seen as a simple cumulative proxy for the actual content of individual messages. The key results are illustrated in Fig. 5.a) and Fig. 5.b) which show the 100 most frequent words in respectively the ASD and control data sets, displayed as so-called 'word-clouds' whereby the frequency of a particular word is encoded 
by the corresponding font size. We used a linear scale, the font size thus being proportional to the corresponding word's frequency in a data set.

(a) ASD 'word-cloud'

(b) Control 'word-cloud'

Fig. 5: The most frequent words in the (a) ASD and (b) control data sets, shown as socalled 'word-clouds'. The font size used to display a particular word is proportional to the corresponding word's frequency in the data set.

As readily observed from Fig. 5 the most commonly used words in the two groups of tweets reveal a substantial difference in the nature of discussed topics. A more thorough examination reveals even further meaningful patterns coherent with the existing literature on ASD. In particular, observe the presence of a large number of words in the plot corre- 
sponding to the ASD group which are related to children such as "children", "kids", "child", and "son", for example. There are a number of reasons why this is unsurprising. Firstly, the diagnosis of ASD is usually made in early childhood so it is reasonable to expect that the parents confronting this new challenge would have increased initiative at seeking help from the community of parents in a similar situation. In addition, while undoubtedly always vulnerable, the vulnerability of individuals on the autism spectrum is the greatest while they are young which is when they need the most support from their guardians and therapists e.g. at acquiring the skills needed to progress thorough the educational system and integrate in the society.

It is also interesting to observe the high frequency of the words "son" and "boy" in the ASD data (top-right section of the word cloud), and the absence of the equivalent female sex words "daughter" and "girl". This example illustrates well how the content of tweets can be used to extract some rather subtle information. In particular in this case our findings are consistent with the understanding of the medical community and a body of evidence which shows that boys are nearly five times more likely to suffer from autism than girls [39].

In contrast, the most frequently used words in the control group do not seem to follow any particular pattern or focus of interest, and instead pertain to more general everyday interests and activities. Lastly, it is interesting to observe that the set of words in Fig. 5(a) appears to contain more nouns than Fig. 5 (b), and fewer verbs. This suggests a different nature of information exchange in the ASD group which appears more focused on issues (syndromes, disorders, interventions, support, help, and so on) and individuals (mostly children), both being described by nouns, while the users who posted tweets of the control group seem to be more interested in what it is that they are or will be doing (like, want, go, think, and so on). We will explore this quantitatively in more detail in Section 4.4 .3

\subsubsection{Hashtag analysis}

An alternative proxy for tweet content can be found in user-designated tags, the so-called 'hashtags'. These can be recognized by the leading special character '\#' (the hash sign) and are by convention understood to be meta-data labels in some manner connected with 
the content of the tweet which contains them. Recall from Section 4.1 that 2,260,284 or approximately $25 \%$ of the collected tweets contain hashtags.

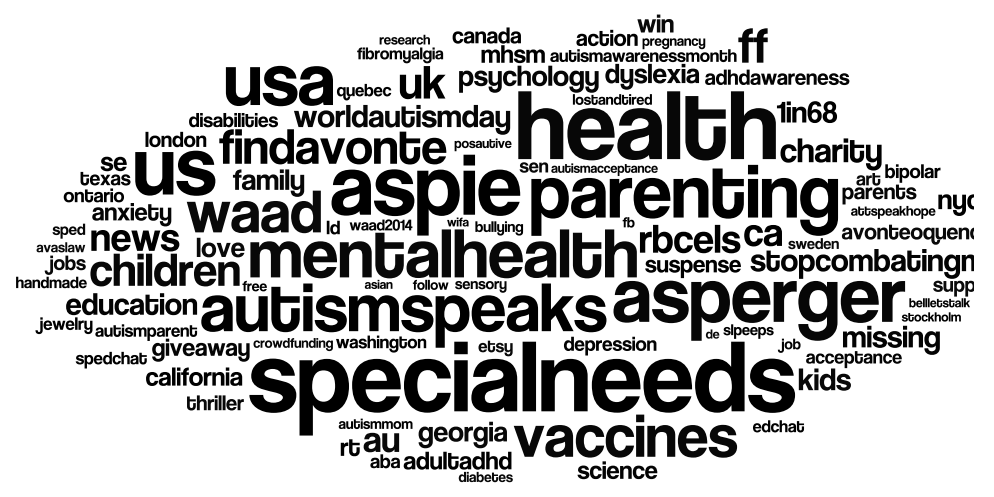

(a) ASD hashtags 'word-cloud'

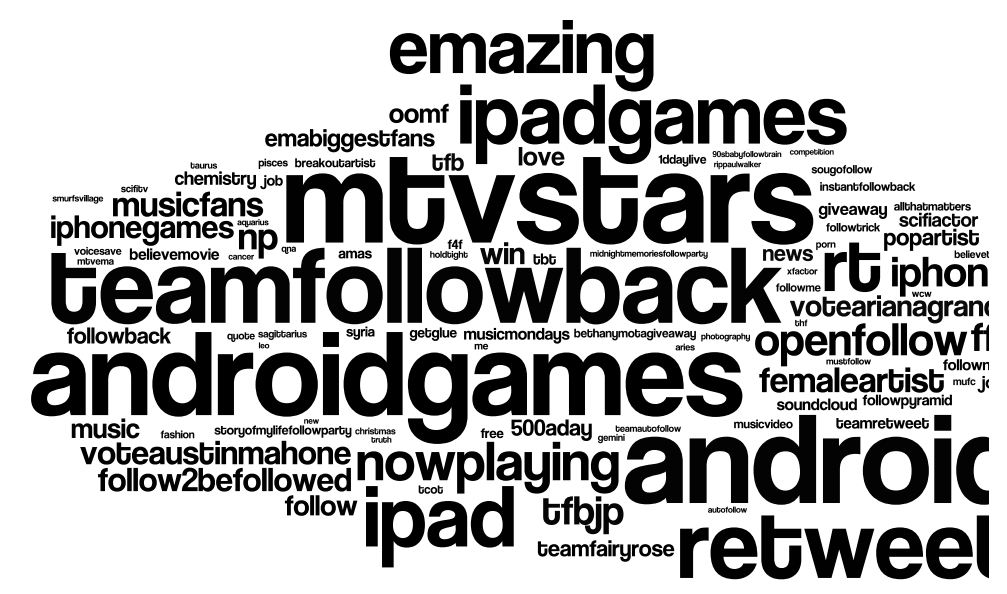

(b) Control hashtags 'word-cloud'

Fig. 6: The most frequent hashtags in the (a) ASD and (b) control data sets, shown as socalled 'word-clouds'. The font size used to display a particular word is proportional to the corresponding word's frequency in the data set. Note that the hashtags corresponding to the search keywords have been removed before producing the ASD word-cloud. 
We summarize our findings as word-clouds of the 100 most frequent hashtags in the ASD and control data sets in Fig. 6,a) and Fig. 6. b). As in the previous analysis of the most frequently used terms, the difference between the two data sets is readily apparent. Unsurprisingly, most of the hashtags in the ASD data set pertain to health issues, some of which are ipso facto ASD-related, such as "mentalhealth" and "psychology", while others are less obviously so. Examples of the latter include "fibromyalgia" and "vaccines". Fibromyalgia is a class of disorders related to the body's processing of pain which recent evidence suggests may have a potential connection with ASD [40]. Similarly, although now discredited, previous research had suggested a causal link between children being vaccinated and developing autism [41]. These examples are further evidence of the type of powerful information which can be harvested from Twitter. In particular, it shows that it is possible to data-mine tweets to provide feedback to medical practitioners on the concerns of the ASD community, the penetration (or lack thereof) of relevant public health recommendations (e.g. ABA or the applied behaviour analysis), or the adoption of treatments of questionable efficacy (e.g. homeopathy, gluten-free diet).

Much like the word-cloud in Fig. 5.a), the hashtag word-cloud in Fig.6.a) contains many references to various topics pertaining to the support of individuals on the autism spectrum. However, the nature of the two sets of terms is somewhat different. While the most frequently used terms mostly referred to the issues themselves, the corresponding hashtags mostly refer to particular support groups. For example, "autismspeaks" (centre-left position within the word-cloud) is a US-based autism advocacy organisation, "thisisautism" (centreright position within the word-cloud) a Twitter stream used to share autism-related experienced, and 'AS2DC' (top-right corner of the word-cloud) an action summit on autism held in Washington D. C.

\subsubsection{Part-of-speech analysis}

In Section 4.4.1, in the discussion of our findings in the comparative analysis of the most frequently used words in the ASD and control tweets, we observed that the ASD tweets appeared to contain a greater proportion of nouns and a smaller proportion of verbs than 
the control tweets. This observation led us to investigate this matter in further detail. In particular, we compared the usage of different part-of-speech (POS) types in the two data sets.

We used the free TweetNLP software package [42], trained on Twitter data, to tag automatically all terms in our entire data set according to their POS type. A selected set of the most interesting results is shown in the plots in Fig. 7. The first thing to observe from the entire corpus of results is that there is a consistent difference between the ASD and control tweets. Notwithstanding the large standard deviation values and the significant overlap of distributions corresponding to ASD and control tweets, that the difference between the two corpora is real rather than a result of stochasticity, is readily witnessed by the fact that in each of the plots the same relative behaviour is exhibited regardless of the tweet length. We confirmed this statistical significance rigorously too. In order to collate all data shown in a single plot, rather than considering the actual POS type count per tweet we considered POS type count normalized by tweet length. This allowed us to perform a single Student's $t$-test per POS type (i.e. plot in Fig. 7). In all cases we obtained $p<0.05$.

Next we turned our attention to the interpretation of the specific results in Fig. 7 Note by observing the plots in Fig. 7 a) and Fig. 7 b) that our hypothesis from Section 4.4.1 regarding the type of information communicated within the ASD and control sub-groups, is confirmed. Recall that by examining qualitatively word clouds of the dominant terms in the two corpora we hypothesised that the tweets in the ASD sub-group are more focused on issues (syndromes, disorders, interventions, support, help, and so on) and individuals (mostly children), described by nouns, while the control tweets more often relate to what it is that their authors are doing (liking, wanting, going, thinking, etc). Indeed for the average tweet length (as counted by the number of words in a message) of 15-20 terms, the number of nouns in the ASD data set is about twice the number of nouns in the control data set. The difference in the number of verbs, while not as substantial, is also significant - for the average length message, the control data set contains approximately $20 \%$ more verbs. Interestingly, the number of proper nouns does not appear to differ significantly between the two groups. This is perhaps somewhat surprising, as it could be expected that the control 
group would engage more in the discussion of so-called celebrities. A more thorough, manual examination of relevant tweets reveals the reason - while the ASD group does engage in less chatter about celebrities, this is offset by the substantial attention that autistic people, specific autism activists, or politicians commenting or acting on autism-related issues receive.

\subsection{Tweet classification}

In Section 4.2.1 we provided evidence that tweets, including those made by the community interested in the ASD, obey some of the same general regularities when it comes to the pattern of word usage. Then, starting in Section 4.3 and corroborating this further in Sections 4.4.1, 4.4.2 and 4.4.3 we showed that in terms of their semantics and content, messages of the ASD community appear to exhibit a range of characteristics which differentiate them from those in our control data set. This motivated us to explore if it is possible to classify tweets automatically as belonging to the ASD data set or not.

It is important to emphasise right at the beginning that in this experiment we eliminated from all tweets the five search keywords which we used to divide our entire tweet data corpus into ASD and control sets i.e. which we used to define the quasi-ground truth labelling. Recall from Section 4.1 that these keywords are "autism", "adhd", "asperger", and "aspie". Had they not been removed, classification performance would have been artificially increased as their presence in a tweet would have been learnt as being capable of perfectly predicting classification output. Furthermore, it is reasonable to expect that the 'true' ASD corpus of tweets should include some tweets which do not contain any of the aforementioned five keywords. In that sense, we hypothesised that it would be possible to use simple tweet filtering based on a small number of obvious keywords to build a bootstrap training data set which could be in turn used to learn additional aspects characterizing ASD-related tweets, thereby facilitatating a more robust retrieval of messages of interest.

Discriminative power of individual terms To examine the discriminative potential of tweet content following the removal of our keywords, we first looked at the discriminative power 


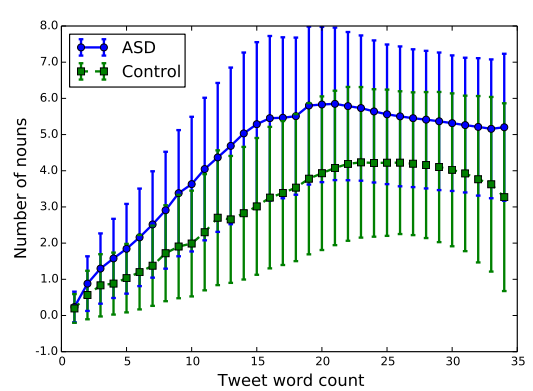

(a) Nouns

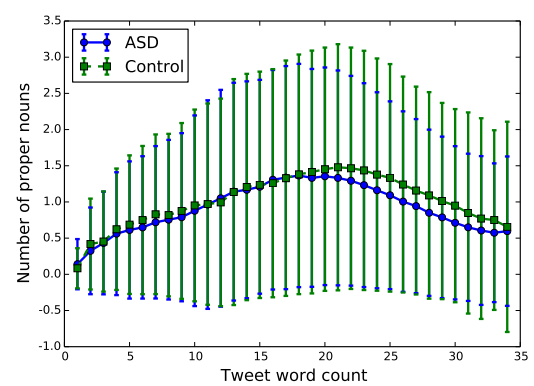

(c) Proper nouns

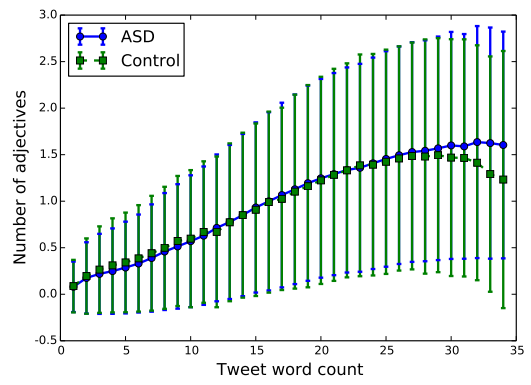

(e) Adjectives

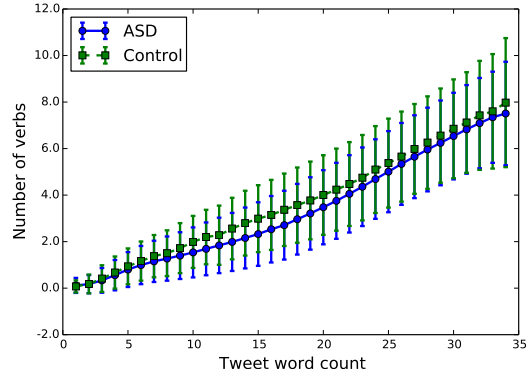

(b) Verbs

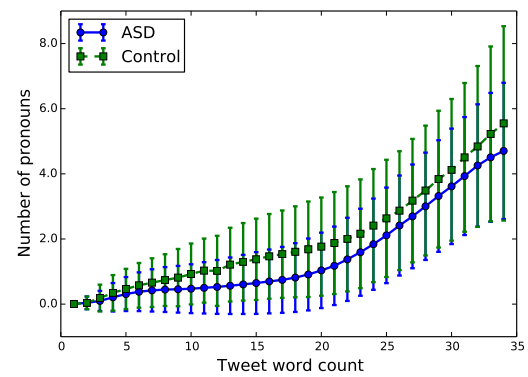

(d) Pronouns

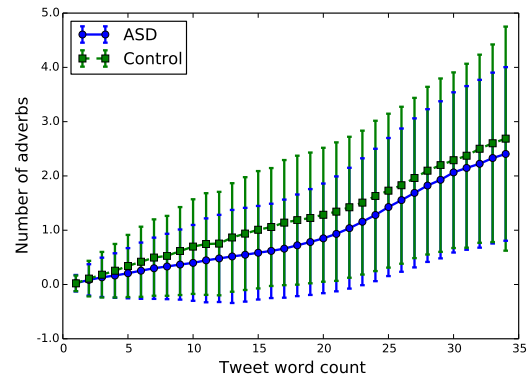

(f) Adverbs

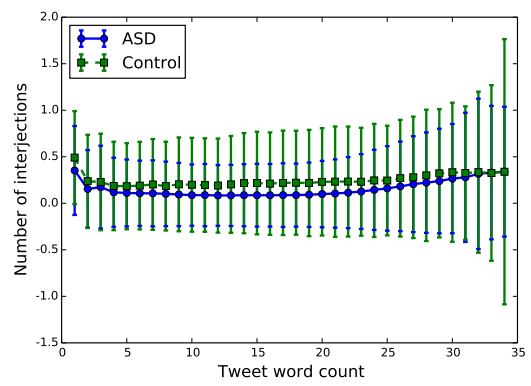

(g) Interjections

Fig. 7: A summary of the key results of comparative part-of-speech analysis. In all cases a statistically significant $(p<0.05)$ difference between ASD and control sub-groups is observed. The most interesting insight is that tweets in the ASD sub-group are more focused on issues (syndromes, disorders, interventions, support, help, and so on) and individuals (mostly children), described by nouns, while the control tweets more often relate to what it is that their authors are doing (liking, wanting, going, thinking, etc). 
of individual words. This is effectively visualized in Fig. 8. What the plot shows is the most frequent 1300 words in our entire data set, which covers respectively $75 \%$ and $83 \%$ of the words in ASD and control subsets, depicted as circular blobs and colour-coded by their discriminative power (specifically, the saturation of a blob's colour is proportional to the absolute value of the logarithm of the ASD-control likelihood ratio of the corresponding word). As expected based on our previous results presented in Sections 4.4.1 and 4.4.2, a number of words pertaining to issues of most concern to the ASD community are highly discriminative. This observation motivated our next set of experiments which examined tweet classification in detail.

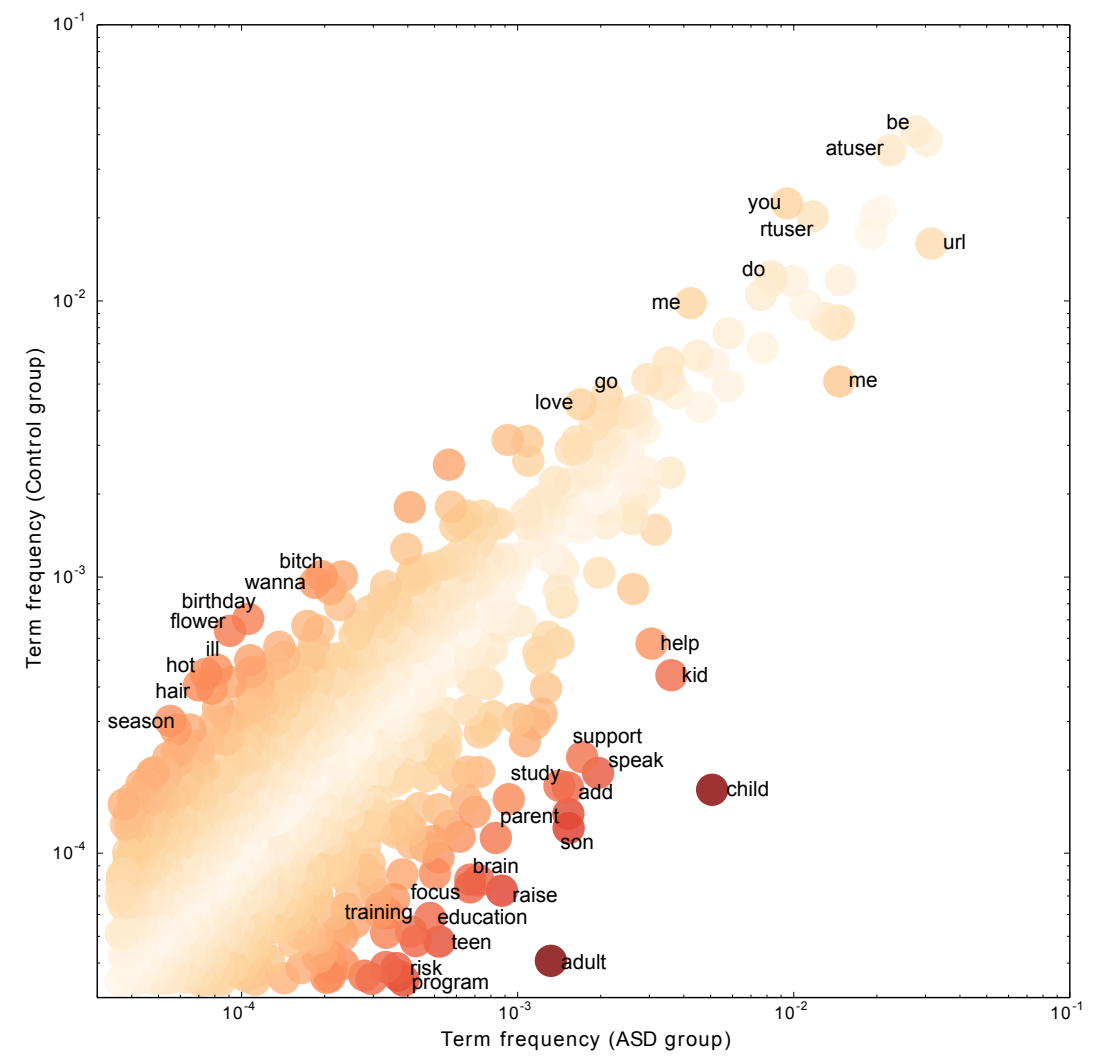

Fig. 8: A compact illustration of the discriminative power of different individual words. Each circular blob represents a word, the saturation of its colour being proportional to the absolute value of the logarithm of the ASD-control likelihood ratio of the word. 


\subsubsection{Automatic classification}

Representations We evaluated a number of different representations of tweet content. Here we present three which overall produced the most interesting results. These are variations on well-known representations in the existing literature, adapted to the problem at hand. Specifically, we report on the performance of the following:

- binary bag of terms (pre-processed words) [43, 44],

- integer bag of terms (also referred to as term count) [45], and

- tf-idf (term frequency-inverse document frequency) score [46].

In the binary bag of terms representation used in this work, each entry $x_{i}$ in a feature vector $\mathbf{x}_{\text {bow }}=\left[x_{1}, x_{2}, \ldots, x_{n_{w}}\right]^{T}$ corresponds to a particular term and is coded as either present in a particular tweet (value 1) or absent from it (value 0), where $\mathbf{x}_{\text {bow }} \in \mathbb{R}^{n_{d}}$ and $n_{d}$ is the dictionary size over which the feature vector is constructed (we will discuss vocabulary construction in more detail shortly). Thus, the original tweet:

Looks like we will have more \#autism research happening for children in \#EarlyIn-

tervention next year! :-) \#VisualSupports \#MobileTech

which following our pre-processing ends up as:

\section{look like autism research happen child in earlyintervention visualsupports mobiletech}

is represented using the following binary bag of words feature vector:

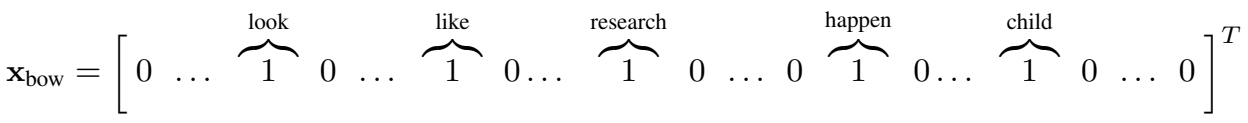

Note that the term "autism" is not included as it is matched by one of our search keywords used for quasi-ground truth labelling, as described in Section 4.1. On the other hand, the terms "earlyintervention", "visualsupports", and "mobiletech" do not have the corresponding entries in the feature vector because their frequency across the data corpus is too low 
i.e. they are not amongst the most frequent terms included in our vocabulary. Lastly, "in" is excluded (automatically, as all others) on the basis that it is too short.

The term count representation is similar to the binary bag of words described previously. As before each entry in a feature vector corresponds to a particular term. However, unlike before the entries are not binary variables which signify the presence or the absence of a particular term but rather actual counts of the term's instances in a tweet. Since the previously given example of a tweet does not include any repeated terms, the term count feature vector $\mathbf{x}_{\mathrm{tc}} \in \mathbb{R}^{n_{d}}$ in this instance is identical to $\mathbf{x}_{\mathrm{bow}}$. On the other hand, the tweet mentioned in Section 4.1.1 which after pre-processing looks as follows:

101 autism genet analysi individu autism find gene delet use power genet sequenc

url

produces different bag of words and term count feature vectors, the former having the value of 1 for the entry corresponding to the term "genet" and the latter the value of 2 since the term has two occurrences in the tweet.

Lastly, the tf-idf representation $\mathrm{x}_{\mathrm{t} f i d f} \in \mathbb{R}^{n_{d}}$ too has entries which correspond to different vocabulary terms, but with values which measure the importance of a particular term in a tweet. Specifically, a particular entry is equal to the frequency of the corresponding term ('term frequency') weighted by the inverse number of tweets in the training corpus which also contain the term ('document frequency'). This representation has been used successfully in a variety of applications, from text mining [46] to visual object recognition and retrieval [47].

Vocabulary construction We also explored a number of different ways of selecting the vocabulary over which tweet feature vectors are constructed based on the discriminativeness of terms, as well as their group-based or combined frequencies of use. Specifically, we examined vocabularies formed by choosing the most frequent terms in one of the two sub-groups, the most discriminative terms in one of the two sub-groups (as explained in Section 4.5 and visualised in Fig. 8, the union or the intersection of either of the former (e.g. the union of the most discriminative terms in the ASD sub-group and the most discriminative words in the 
control sub-group), the most frequent words across the entire corpus (i.e. both sub-groups), and the most discriminative words across the entire corpus. Our results suggest that the best performance is achieved by selecting a set of the overall most frequent terms; due to the limitations of space we report the corresponding results only, using the 1500 most frequent terms.

Classification methodology In all experiments reported in this section we adopt the supervised classification paradigm. Specifically, we assume that we have available a training set of pairs $\left\{\left(\mathbf{x}_{1}^{(t)}, y_{1}^{(t)}\right),\left(\mathbf{x}_{2}^{(t)}, y_{2}^{(t)}\right), \ldots,\left(\mathbf{x}_{n_{t}}^{(t)}, y_{n_{t}}^{(t)}\right)\right\}$ where $\mathbf{x}_{i}^{(t)}$ is the feature vector (representation) corresponding to the $i$-th training tweet and $y_{i}^{(t)}$ its binary label signifying if the tweet belongs to the ASD or the control sub-group. After a classifier is trained the label of a novel query tweet described by the feature vector $\mathbf{x}$ is performed as follows:

$$
y=\arg \max _{y} \frac{\operatorname{Pr}(y) \operatorname{Pr}(\mathbf{x} \mid y)}{\operatorname{Pr}(\mathbf{x})}=\arg \max _{y} \operatorname{Pr}(y) \operatorname{Pr}(\mathbf{x} \mid y)
$$

In our experiments we used half of the collected data corpus for training, and the remaining half for testing the performance of different representations and classifiers. Three popular classification methods were examined:

- naïve Bayes-based [48],

- logistic regression-based [49], and

- least absolute shrinkage and selection operator (LASSO)-based.

In naïve Bayes classification the strong assumption of independence between different terms in a feature vector is assumed resulting in the following class likelihood estimate:

$$
P_{N B}(y= \pm 1 \mid \mathbf{x}) \propto \prod_{i=1}^{n_{w}} \operatorname{Pr}\left(x_{i} \mid y= \pm 1\right)
$$

where, without loss of generality, the values +1 and -1 of the dependent variable $y$ are used to signify respectively ASD and control sub-group memberships. Conditional probabilities 
$\operatorname{Pr}\left(x_{i} \mid y= \pm 1\right)$ are estimated in a straightforward manner from the frequencies of the corresponding terms in the training data corpus.

In logistic regression, the conditional probability of the dependent variable $y$ is modelled as a logit-transformed multiple linear regression of the explanatory variables $x_{1}, \ldots, x_{n_{e}}$ :

$$
P_{L R}(y= \pm 1 \mid \mathbf{x}, \mathbf{w})=\frac{1}{1+e^{-y \mathbf{w}^{T} \mathbf{x}}}
$$

The model is trained (i.e. the weight parameter $\mathbf{w}$ learnt) by maximizing the likelihood of the model on the training data set, given by:

$$
\prod_{i=1}^{n_{t}} \operatorname{Pr}\left(y_{i}^{(t)} \mid \mathbf{x}_{i}^{(t)}, \mathbf{w}\right)=\prod_{i=1}^{n_{t}} \frac{1}{1+e^{-y_{i}^{(t)} \mathbf{w}^{T} \mathbf{x}_{i}^{(t)}}}
$$

penalized by the complexity of the model:

$$
\frac{1}{\sigma \sqrt{2 \pi}} e^{-\frac{1}{2 \sigma^{2}} \mathbf{w}^{T} \mathbf{w}}
$$

which can be restated as the minimisation of the following regularized negative log-likelihood:

$$
\mathfrak{L}=C \sum_{i=1}^{n_{t}} \log \left(1+e^{-y_{i} \mathbf{w}^{T} \mathbf{x}_{i}}\right)+\mathbf{w}^{T} \mathbf{w} .
$$

A coordinate descent approach described by Yu et al. [49] was used to minimize $\mathfrak{L}$.

Finally, in LASSO-based classification, sparseness of the solution is achieved by replacing the $L_{2}$-norm penalty in 6 with $L_{1}$-norm, yielding the following regularized negative log-likelihood:

$$
\mathfrak{L}=C \sum_{i=1}^{n_{t}} \log \left(1+e^{-y_{i} \mathbf{w}^{T} \mathbf{x}_{i}}\right)+\lambda\|\mathbf{w}\|_{1} .
$$

where $\lambda$ is a free parameter governing the tradeoff between prediction loss and solution sparsity.

Basic classification: results and discussion We first evaluated the naïve Bayes and logistic regression-based classifiers as these do not have free parameters i.e. parameters which must 
be set a priori. The corresponding classification results are summarized in Table 1 With the exception of the result achieved with logistic regression and the tf-idf representation, different combinations of base classifiers and representations performed nearly identically. Specifically, it can be seen that approximately $79 \%$ of tweets were correctly classified. This is a rather remarkable performance considering the brevity of tweets and the fact that some of the most informative (in the context of the classification task at hand) words were not used for classification. It is insightful to notice the consistently higher accuracy attained for control (84-85\%) rather than ASD tweets (71-74\%). We explored this finding in more depth by manually inspecting misclassified messages. Within the ASD data corpus, we found that the main source of classification error lied in the absence of any ADS-specific information in very short tweets, after the removal of our four keywords used to construct the data set in the first place (e.g. "it is the adhd, oops!"). This is a highly comforting finding since of course in any practical application these keywords would not be eliminated, thus improving classification performance dramatically.

Table 1: A summary of the classification results (ASD-related vs. non-ASD-related tweets). Each cell (corresponding to a combination of a classification method and a representation) shows the associated confusion matrix. The first row/column of a confusion matrix corresponds to the control class and second row/column to the ASD class (thus for e.g. using the term count representation, naïve Bayes correctly correctly classified $84 \%$ of control tweets and $71 \%$ of ASD tweets).

\begin{tabular}{|l|c|c|c|}
\cline { 2 - 4 } \multicolumn{1}{c|}{} & \multicolumn{3}{c|}{ Confusion matrix } \\
\hline Representation & Binary & Term count & tf-idf \\
\hline \multirow{2}{*}{ Naïve Bayes } & 0.840 .16 & 0.840 .16 & 0.840 .16 \\
& 0.290 .71 & 0.290 .71 & 0.380 .62 \\
\hline \multirow{2}{*}{ Logistic regression } & 0.850 .15 & 0.850 .15 & 0.030 .97 \\
& 0.260 .74 & 0.270 .73 & 0.040 .96 \\
\hline
\end{tabular}


We also experimented with the application of latent semantic analysis (LSA) [50], testing out the possibility that better discrimination may be achieved by working in the so-called concept rather than term space. Although LSA has been shown to be highly successful in a variety of information retrieval tasks, our experiments suggest that the same benefit is not observed when it is applied on tweets. The likely reason for this can be found in the brevity of tweets, which favours the use of succinct expressions whereby individual terms (unigrams) themselves effectively become LSA concepts. This also explains why the simple naïve Bayes classifier performed on par with the generally superior logistic regression-based one, as can be seen in Table 1

We next evaluated the LASSO-based classifier. In order to examine fully the behaviour of this approach we did not perform the usual procedure for selecting the value of the free parameter $\lambda$ using cross-validation; rather we measured classification performance across a range of $\lambda$ values. Specifically we considered values from $\lambda=10 e^{-5}$ up to $\lambda=10 e^{-2}$; greater values than $10 e^{-2}$ produced numerical problems caused by an excessively small set of selected predictor terms. Our results are summarized in Fig. 9 The plot shows that the classifier exhibits consistent performance across the range of $\lambda$ between $10 e^{-5}$ and $10 e^{-4}$, with the accuracy of the classification of ASD tweets deteriorating thereafter (with a small increase in the accuracy of control tweet classification). This deterioration is readily explained by examining the number of terms selected by LASSO i.e. by the number of terms with non-zero corresponding regression coefficients. For $\lambda=10 e^{-5}$ this number is 801 , dropping down for $\lambda=10 e^{-4}$ to 580 , and reducing drastically to 151 for $\lambda=10 e^{-3}$ and to 12 for $\lambda=10 e^{-2}$ as a consequence of the harsh penalty on model complexity effected by the large values of $\lambda$.

Lastly, useful insight can be gained by considering the terms which correspond to the largest magnitude regression coefficients. These are, in order, "help", "kid", "aw" (the automatically constructed stem of words such as "awareness" and "aware"), and "child". The corresponding regression coefficients are all positive i.e. the presence of these terms predicts ASD-related tweets. Consequently, control tweets are predominantly predicted by the absence rather than presence of specific terms; the highest magnitude negative coefficient, 


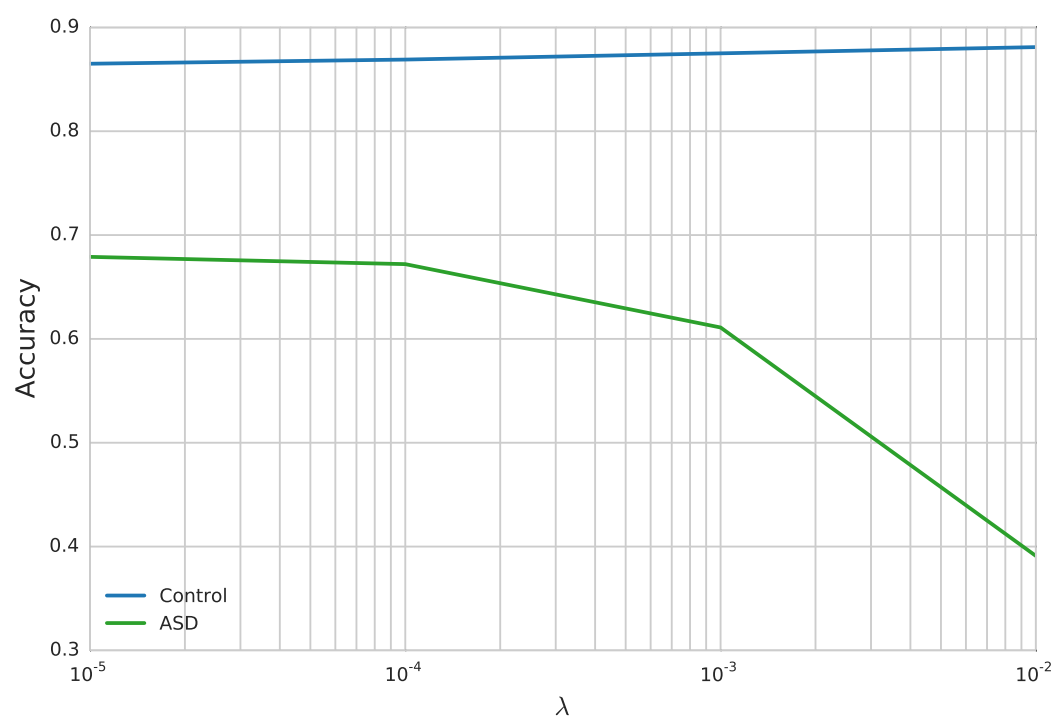

Fig. 9: LASSO-based classification performance as a function of the loss-sparseness tradeoff parameter $\lambda$. The number of non-zero regression coefficients for $\lambda$ equal to $10 e^{-5}, 10 e^{-4}$, $10 e^{-3}$, and $10 e^{-2}$ is respectively $801,580,151$, and 12 . High values of $\lambda$ which severely penalize model complexity can be seen to result in deteriorating classification performance for ASD-related tweets.

that is coefficient which corresponds to the term which predicts a control tweet, is "follow". The observation that control tweets are predicted by the absence of ASD-related terms explains why a reduction in model complexity (i.e. a reduction in the number of ASD-terms considered by the classifier) does not produce a deterioration in classification performance on control tweets (rather, a small improvement can be noticed) while a major negative effect is readily observed for the ASD corpus.

Condition-oriented classification: results and discussion To motivate our next set of experiments, consider a classifier which distinguishes between medical condition-related and other tweets. Let the classifier correctly classify a tweet of the former type (dependent variable value +1 ) with the probability $p_{m}$ and of the latter type with the probability $p_{n}$ (dependent variable value -1 ). Imagine applying this classifier on our data set but interpreting its output 
as classifying tweets as being ASD related $(+1)$ or not $(-1)$. Since all ASD tweets are medical condition related, the probability of the classifier output being +1 given an ASD tweet is also $p_{m}$. On the other hand, the control corpus contains both tweets not related to a medical condition as well as those which are related to medical conditions other than ASD. Hence, the probability of the classifier output being -1 given a tweet from our control corpus is:

$$
p_{n}\left(1-\rho_{m}\right)+\left(1-p_{m}\right) \rho_{m}
$$

where $\rho_{m}$ is the proportion of control tweets which are medical condition-related. The first term, $p_{n}\left(1-\rho_{m}\right)$, is the probability of a control tweet not related to a medical condition being selected as input to the classifier (probability $1-\rho_{m}$ ) and correctly classified (probability $\left.p_{n}\right)$. The second term, $\left(1-p_{m}\right) \rho$, is the probability of a medical condition related control tweet being selected as input (probability $\rho_{m}$ ) and incorrectly classified (probability $\left.1-p_{m}\right)$, as the classifier output of interest is -1 . The expression in 8 can be rearranged to give:

$$
p_{n}+\left(1-p_{m}-p_{n}\right) \rho_{m}
$$

Since the proportion $\rho_{m}$ of medical condition related tweets is likely to be small, it can be seen that a good classifier which differentiates between medical condition related and other tweets can appear to be a good classifier of ASD vs. non-ASD-related tweets. Thus the question is if the results of our experiments described in the previous section really demonstrate good ASD vs. non-ASD discrimination, or is it simply the case that our methods learnt to distinguish between medical condition-related and non-medical condition-related tweets. We set out to investigate this next.

For this experiment we followed the methodology described in the previous set of experiments with the sole difference being that learning was done using a different control corpus. Specifically, we collected a data set related to another pervasive medical condition - Alzheimer's disease. In a similar manner as in Section 4.1 we collected tweets which contain the word "alzheimer" (or any of the words derived from it by suffixation), balancing 
their number with the number of ASD-related tweets in our data set. Considering the little difference in performance obtained by the use of different representations in our previous set of experiments, we did not examine them all here too; instead we adopted the term count representation as the most commonly used one in the literature.

Our results are summarised in Table 2 . It can be readily observed, especially in the context of the evidence presented previously, that this experiment too strongly supports our thesis that the content of ASD tweets, even with the most salient words removed (the keywords used to collect the corpus as described in Section 4.1 is highly informative. Both Alzheimer's disease-related and ASD-related tweets were classified with nearly perfect accuracy. As in our previous experiments there was little difference between the performance of the naïve Bayes classifier and that based on logistic regression, the latter achieving somewhat better results on ASD-related tweets (100\% vs. 98\% accuracy).

Table 2: A summary of the classification results (ASD-related vs. Alzheimer disease-related tweets). Each cell shows the associated confusion matrix. The first row/column of a confusion matrix corresponds to the Alzheimer's disease class and second row/column to the ASD class.

\begin{tabular}{|l|c|}
\cline { 2 - 2 } \multicolumn{1}{c|}{} & Confusion matrix \\
\hline Representation & Term count \\
\hline \multirow{2}{*}{ Naïve Bayes } & 0.980 .02 \\
& 0.050 .95 \\
\hline \multirow{2}{*}{ Logistic regression } & 0.980 .02 \\
& 0.001 .00 \\
\hline
\end{tabular}

4.6 Bootstrap keyword set analysis

Recall from Section 4.1 that we collected the ASD tweet corpus by including in it any tweet which contained any of the four keywords "autism", "adhd", "asperger", and "aspie" (or 
any of their derivatives obtained by suffixation). Moreover, in all experiments in which the difference between tweets related and unrelated to ASD was examined, the ground truth was defined by the presence of these words. This was necessitated by the scale of the data which made it practically impossible to perform labelling manually. Consequently, these keywords had to be excluded from the consideration in classification experiments. Clearly this would not be done in any practical application which means that the results we presented are worse, likely significantly so, than they would have been had we been able to use the entirety of tweet content. The high classification accuracy we obtained of approximately $80 \%$ provides strong evidence that even with these most salient words removed, the remaining content of ASD-related tweets is highly informative and characteristic. In the final set of experiments we report here we examined the sensitivity of learning to the exact keywords used to select the learning corpus.

We repeated the classification experiments from the previous section but using for training only a part of the previous training corpus - the part that is matched by three of the four keywords. Thus four experiments were performed, with one of the keywords being left out in each. Then we first evaluated the performance of thus learnt classifier on the test corpus, just as before. We additionally evaluated the performance of the classifier specifically on those tweets which contained the left out keyword but none of the other three. Our results are summarized in the bar plot in Fig. 10. Firstly, observe that in no experiment was the classification performance on the test set greatly negatively affected - in all cases $83-85 \%$ of control tweets were correctly classified and $71-76 \%$ of ASD-related tweets. This is in line with our previous observations and is highly reassuring since it suggests that the same type of characteristic context is learnt regardless of which specific keywords are used to collect training data. However, the classification results on the tweets only matched by the left out keyword is interesting and provides novel insight. In particular, observe the particularly bad performance in the experiment in which the keyword "adhd" was left out - only about $44 \%$ of the tweets matched by this keyword were correctly classified. A manual examination of the misclassified tweets readily revealed the answer - in nearly all cases these seemingly 
misclassified tweets indeed were not related to ASD. Rather the term "adhd" was used as a hyperbole for an inattentive or easily distracted person. Here are a few examples:

- Watching football is such a commitment. I'm too adhd for this.

- I think we both have adhd

- Ifeel like I have adhd.. I cannot write 300 words and not stop for a "break"

- $\quad$ And then your adhd causes you to forget everything as soon as you get on your phone

We made a similar observation with regard to the usage of the word "aspie" which too is used loosely in colloquial speech (albeit with a lower frequency than "adhd"). This explains the performance of the classifier trained on data matched by the other three keywords and evaluated on the tweets matched by "aspie" only, which was also worse than the corresponding performance in the experiments in which the left out keyword was "autism" or "asperger" but not as much as when "adhd" was left out.

In summary, this experiment offers several important insights useful in the practical application of the ideas presented in the present paper. Firstly, it showed that the choice of keywords does not need to be a complex process in the sense that a small number of keywords is sufficient to learn the relevant salient characteristics of a theme associated with them. Additional keywords appear to be redundant, adding no new information and not resulting in improved performance. Secondly, the experiment demonstrated how potentially bad keyword choices can be automatically detected and therefore removed. This can be done either fully automatically or in a semi-automatic fashion following human input (approval).

\section{Summary and conclusions}

The aim of this work was to investigate the potential of data-mining messages posted on Twitter to learn about the concerns, practices, and more generally topics of conversation of people interested in the autism spectrum disorder. We approached this problem first by harvesting a large data set of tweets each of which we designated as belonging either to the ASD-related subset of the data corpus or the control subset. Using this data set we conducted a series of experiments which analysed both common and differential characteristics 


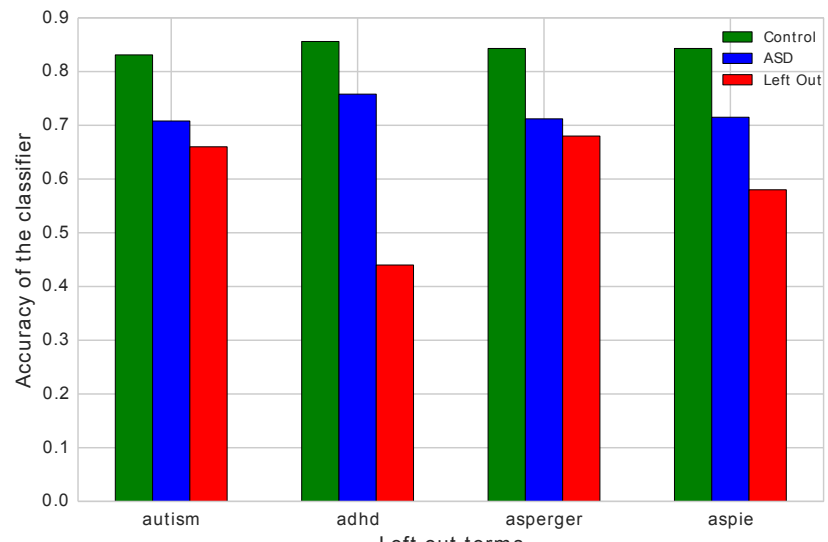

Fig. 10: Classification results using only a subset of the entire training corpus used in the previous section. In each experiment training was performed using tweets matched by the three remaining keywords after one of the original keywords was left out. For each experiment we show three classification accuracies: on (i) test corpus control tweets (green bars), (ii) test corpus ASD-related tweets (blue bars), and (iii) tweets matched by the left out keyword but no others (red). See main text for a discussion of the findings.

of messages in the two subsets and reported a series of novel results. The most important finding, corroborated by several different experiments, concerns the nature of topics arising in the ASD subset of tweets. We demonstrated that tweets in this subset are very rich in information of potentially high value to public health officials and policy makers, thus motivating further work towards our goal of developing a tool which would be able to monitor automatically the response of the ASD community to various initiatives, legislature, medical advances etc. For example, driven by the finding that a high proportion of the tweets in our corpus contain URL links, one of the directions we wish to pursue in future is that of exploring the value of auxiliary information which can be associated with original messages. We also intend to explore automatic ways of labelling topics semantically using meaningful sentence fragments by back-analysing probabilistically the collected text data for persistent ngrams across documents with shared topics [51]. 


\section{Acknowledgements}

The authors would like to express their sincere gratitude to the anonymous reviewers whose constructive feedback on our original work [1] greatly helped shape the present paper. Specifically, we are thankful for their suggestions for additional experiments which were described herein.

\section{References}

1. A. Beykikhoshk, O. Arandjelović, D. Phung, S. Venkatesh, and T. Caelli. Data-mining Twitter and the autism spectrum disorder: a pilot study. In Proc. IEEE/ACM International Conference on Advances in Social Network Analysis and Mining, pages 349-356, 2014.

2. J. H. Miles. Autism spectrum disorders-a genetics review. Genetics in Medicine, 13:278-294, 2011.

3. Autism spectrum disorder fact sheet. American Psychiatric Publishing, 2013.

4. A. J. Baxter, T. S. Brugha, H. E. Erskine, R. W. Scheurer, T. Vos, and J. G. Scott. The epidemiology and global burden of autism spectrum disorders. Psychological Medicine, 45(3):601-613, 2015.

5. A. Beykikhoshk, O. Arandjelović, D. Phung, and S. Venkatesh. Hierarchical Dirichlet process for tracking complex topical structure evolution and its application to autism research literature. In Proc. Pacific-Asia Conference on Knowledge Discovery and Data Mining, 1:550-562, 2015.

6. L. Kanner. Irrelevant and metaphorical language in early infantile autism. American Journal of Psychiatry, 103(2):242-246, 1946.

7. S. E. Levy, D. S. Mandell, and R. T. Schultz. Autism. Lancet, 374(9701):1627-1638, 2009.

8. D. E. Gray. Perceptions of stigma: The parents of autistic children. Sociology of Health \& Illness, 15(1):102-120, 1993. 
9. Z. Warren, M. L. McPheeters, N. Sathe, J. H. Foss-Feig, A. Glasser, and J. VeenstraVanderWeele. A systematic review of early intensive intervention for autism spectrum disorders. Pediatrics, 127(5):e1303-e1311, 2011.

10. J. T. Danial and J. J. Wood. Cognitive behavioral therapy for children with autism: Review and considerations for future research. Journal of Developmental \& Behavioral Pediatrics, 34(9):702-715, 2013.

11. D. Trembath, S. Balandin, and C. Rossi. Crosscultural practice and autism. Journal of Intellectual and Developmental Disability, 4(30):240-242, 2005.

12. J. W. Harrington, L. Rosen, A. Garnecho, and P. A. Patrick. Parental perceptions and use of complementary and alternative medicine practices for children with autistic spectrum disorders in private practice. Journal of Developmental \& Behavioral Pediatrics, 27(2):S156-S161, 2006.

13. L. Jiang, M. Yu, M. Zhou, X. Liu, and T. Zhao. Target-dependent Twitter sentiment classification. In Proc. Annual Meeting of the Association for Computational Linguistics, pages 151-160, 2011.

14. A. Bifet and E. Frank. Sentiment knowledge discovery in Twitter streaming data. In Proc. International Conference on Discovery Science, pages 1-15, 2010.

15. A. Agarwal, B. Xie, I Vovsha, O. Rambow, and R. Passonneau. Sentiment analysis of Twitter data. In Proc. Workshop on Language in Social Media, pages 30-38, 2011.

16. S. Asur and B. A. Huberman. Predicting the future with social media. In Proc. IEEE/ACM International Conference on Web Intelligence and Intelligent Agent Technology, pages 492-499, 2010.

17. E. Baucom, A. Sanjari, X. Liu, and M. Chen. Mirroring the real world in social media: Twitter, geolocation, and sentiment analysis. In Proc. International Workshop on Mining Unstructured Big Data Using Natural Language Processing, pages 61-68, 2013.

18. J. Bollen, H. Mao, and A. Pepe. Modeling public mood and emotion: Twitter sentiment and socio-economic phenomena. In Proc. International Conference on Weblogs and Social Media, pages 450-453, 2011. 
19. L. Mitchell, M. R. Frank, K. D. Harris, P. S. Dodds, and C. M. Danforth. The geography of happiness: connecting Twitter sentiment and expression, demographics, and objective characteristics of place. PLOS ONE, 8(5):e64417, 2013.

20. S. Verma, S. Vieweg, W. J. Corvey, L. Palen, J. H. Martin, M. Palmer, A. Schram, and K. M. Anderson. Natural language processing to the rescue? Extracting "situational awareness" tweets during mass emergency. In Proc. International Conference on Weblogs and Social Media, pages 385-392, 2011.

21. B. Robinson, R. Power, and M. Cameron. An evidence based earthquake detector using Twitter. In Proc. Workshop on Language Processing and Crisis Information, pages 1-9, 2013.

22. T. Sakaki, M. Okazaki, and Y. Matsuo. Earthquake shakes Twitter users: real-time event detection by social sensors. In Proc. International Conference on World Wide Web, pages 851-860, 2010.

23. M. J. Paul and M. Dredze. A model for mining public health topics from Twitter. Health, 11:16-16, 2012.

24. M. J. Paul and M. Dredze. You are what you tweet: analyzing Twitter for public health. In Proc. Internation Conference on Weblogs and Social Media, pages 265-272, 2011.

25. K. W. Prier, M. S. Smith, C. Giraud-Carrier, and C. L. Hanson. Identifying healthrelated topics on Twitter, an exploration of tobacco-related tweets as a test topic. In Proc. International Conference on Social Computing, Behavioral-Cultural Modeling, and Prediction, pages 18-25, 2011.

26. J. M. Robillard, T. W. Johnson, C. Hennessey, B. L. Beattie, and J. Illes. Aging 2.0: health information about dementia on Twitter. PLOS ONE, 8(7):e69861, 2013.

27. D. Scanfeld, V. Scanfeld, and E. L. Larson. Dissemination of health information through social networks: Twitter and antibiotics. American Journal of Infection Control, 38(3):182-188, 2010.

28. J. Jashinsky, S. H. Burton, C. L. Hanson, J. West, C. Giraud-Carrier, M. D. Barnes, and Argyle T. Tracking suicide risk factors through Twitter in the US. Crisis: The Journal of Crisis Intervention and Suicide Prevention, 35(1):51-59, 2014. 
29. I. Himelboim and J. Y. Han. Cancer talk on Twitter: community structure and information sources in breast and prostate cancer social networks. Journal of Health Communication, 19(2):210-225, 2014.

30. A. Culotta. Towards detecting influenza epidemics by analyzing Twitter messages. In Proc. ACM Workshop on Social Media Analytics, pages 115-122, 2010.

31. A. Harshavardhan, A. Gandhe, R. Lazarus, S. H. Yu, and B. Liu. Predicting flu trends using Twitter data. In Proc. IEEE Conference on Computer Communications, pages 702-707, 2011.

32. J. Li and C. Cardie. Early stage influenza detection from Twitter. arXiv preprint, page 1309.7340, 2013.

33. C. Chew and G. Eysenbach. Pandemics in the age of Twitter: content analysis of Tweets during the 2009 H1N1 outbreak. PLOS ONE, 5(11):e14118, 2010.

34. A. T. Newton, A. D. I. Kramer, , and D. N. McIntosh. Autism online: a comparison of word usage in bloggers with and without autism spectrum disorders. In Proc. SIGCHI Conference on Human Factors in Computing Systems, pages 463-466, 2009.

35. J. Perkins. Python Text Processing with NLTK 2.0 Cookbook. Packt Publishing, 2010.

36. L. F. Richardson. Variation of the frequency of fatal quarrels with magnitude. Journal of the American Statistical Association, 43(244):523-546, 1948.

37. J. P. Bouchaud and M Mézard. Wealth condensation in a simple model of economy. Physica A: Statistical Mechanics and its Applications, 282(3):536-545, 2000.

38. A. Clauset, C. R. Shalizi, and M. E. Newman. Power-law distributions in empirical data. SIAM Review, 51(4):661-703, 2009.

39. E. Fombonne. Epidemiology of pervasive developmental disorders. Journal of Pediatric Research, 65(6):591-598, 2009.

40. D. A. Geier, J. K. Kern, G. Davis, P. G. King, J. B. Adams, Young J. L., and Geier M. R. A prospective double-blind, randomized clinical trial of levocarnitine to treat autism spectrum disorders. Journal of Medical Science Monitor, 17(6):PI15-PI23, 2011.

41. A. J. Wakefield, S. H. Murch, A. Anthony, J. Linnell, D. M. Casson, M. Malik, M. Berelowitz, A. P. Dhillon, M. A. Thomson, and P. Harvey. RETRACTED: Ileal- 
lymphoid-nodular hyperplasia, non-specific colitis, and pervasive developmental disorder in children. The Lancet, 351(9103):637-641, 1998.

42. O. Owoputi, B. O’Connor, C. Dyer, K. Gimpel, N. Schneider, and N. A. Smith. Improved part-of-speech tagging for online conversational text with word clusters. In Proc. North American Chapter of the Association for Computational Linguistics Conference on Human Language Technologies, pages 380-390, 2013.

43. D. D. Lewis and M. Ringuette. A comparison of two learning algorithms for text categorization. In Proc. Annual Symposium on Document Analysis and Information Retrieval, 33:81-93, 1994.

44. O. Arandjelović. Prediction of health outcomes using big (health) data. In Proc. International Conference of the IEEE Engineering in Medicine and Biology Society, 2015.

45. O. Arandjelović. Automatic attribution of ancient Roman imperial coins. In Proc. IEEE Conference on Computer Vision and Pattern Recognition, pages 1728-1734, 2010.

46. S. Robertson. Understanding inverse document frequency: On theoretical arguments for IDF. Journal of Documentation, 60(5):503-520, 2004.

47. O. Arandjelović. Object matching using boundary descriptors. In Proc. British Machine Vision Conference, 2012. DOI: 10.5244/C.26.85.

48. C. M. Bishop. Pattern Recognition and Machine Learning. Springer-Verlag, New York, USA, 2007.

49. H.-F. Yu, F.-L. Huang, and C.-J. Lin. Dual coordinate descent methods for logistic regression and maximum entropy models. Machine Learning, 85(1-2):41-75, 2011.

50. S. T. Dumais. Latent semantic analysis. Annual Review of Information Science and Technology, 38(1):188-230, 2004.

51. O. Arandjelović. Reading ancient coins: automatically identifying denarii using obverse legend seeded retrieval. In Proc. European Conference on Computer Vision, 4:317-330, 2012. 